\title{
Joint Entity Linking with Deep Reinforcement Learning
}

\author{
Zheng Fang \\ Institute of Information Engineering, \\ Chinese Academy of Sciences \& \\ School of Cyber Security, University \\ of Chinese Academy of Sciences \\ fangzheng@iie.ac.cn \\ Qian Li \\ University of Technology Sydeney \\ Qian.Li@uts.edu.au
}

\author{
Yanan $\mathrm{CaO}^{*}$ \\ Institute of Information Engineering, \\ Chinese Academy of Sciences \\ caoyanan@iie.ac.cn
}

\author{
Dongjie Zhang \\ Institute of Information Engineering, \\ Chinese Academy of Sciences \\ zhangdongjie@iie.ac.cn
}

\author{
Zhenyu Zhang \\ Institute of Information Engineering, \\ Chinese Academy of Sciences \\ zhangzhenyu1996@iie.ac.cn
}

\author{
Yanbing Liu \\ Institute of Information Engineering, \\ Chinese Academy of Sciences \\ liuyanbing@iie.ac.cn
}

\begin{abstract}
Entity linking is the task of aligning mentions to corresponding entities in a given knowledge base. Previous studies have highlighted the necessity for entity linking systems to capture the global coherence. However, there are two common weaknesses in previous global models. First, most of them calculate the pairwise scores between all candidate entities and select the most relevant group of entities as the final result. In this process, the consistency among wrong entities as well as that among right ones are involved, which may introduce noise data and increase the model complexity. Second, the cues of previously disambiguated entities, which could contribute to the disambiguation of the subsequent mentions, are usually ignored by previous models. To address these problems, we convert the global linking into a sequence decision problem and propose a reinforcement learning model which makes decisions from a global perspective. Our model makes full use of the previous referred entities and explores the long-term influence of current selection on subsequent decisions. We conduct experiments on different types of datasets, the results show that our model outperforms state-of-the-art systems and has better generalization performance.
\end{abstract}

\section{CCS CONCEPTS}

\section{- Information systems $\rightarrow$ Information extraction.}

\section{KEYWORDS}

Entity linking, reinforcement learning, joint disambiguation, knowledge base

\section{ACM Reference Format:}

Zheng Fang, Yanan Cao, Dongjie Zhang, Qian Li, Zhenyu Zhang, and Yanbing Liu. 2019. Joint Entity Linking with Deep Reinforcement Learning. In Proceedings of the 2019 World Wide Web Conference (WWW'19), May

\footnotetext{
${ }^{*}$ Corresponding author.
}

This paper is published under the Creative Commons Attribution 4.0 International (CC-BY 4.0) license. Authors reserve their rights to disseminate the work on their personal and corporate Web sites with the appropriate attribution.

WWW '19, May 13-17, 2019, San Francisco, CA, USA

(C) 2019 IW3C2 (International World Wide Web Conference Committee), published under Creative Commons CC-BY 4.0 License.

ACM ISBN 978-1-4503-6674-8/19/05.

https://doi.org/10.1145/3308558.3313517
13-17, 2019, San Francisco, CA, USA. ACM, New York, NY, USA, 10 pages. https://doi.org/10.1145/3308558.3313517

\section{INTRODUCTION}

Entity Linking (EL), which is also called Entity Disambiguation (ED), is the task of mapping mentions in text to corresponding entities in a given knowledge Base (KB). This task is an important and challenging stage in text understanding because mentions are usually ambiguous, i.e., different named entities may share the same surface form and the same entity may have multiple aliases. EL is key for information retrieval (IE) and has many applications, such as knowledge base population (KBP), question answering (QA), etc.

Existing EL methods can be divided into two categories: local model and global model. Local models concern mainly on contextual words surrounding the mentions, where mentions are disambiguated independently. These methods are not work well when the context information is not rich enough. Global models take into account the topical coherence among the referred entities within the same document, where mentions are disambiguated jointly. Most of previous global models [14, 27, 37] calculate the pairwise scores between all candidate entities and select the most relevant group of entities. However, the consistency among wrong entities as well as that among right ones are involved, which not only increases the model complexity but also introduces some noises. For example, in Figure 1, there are three mentions "France", "Croatia" and "2018 World Cup", and each mention has three candidate entities. Here, "France" may refer to French Republic, France national basketball team or France national football team in KB. It is difficult to disambiguate using local models, due to the scarce common information in the contextual words of "France" and the descriptions of its candidate entities. Besides, the topical coherence among the wrong entities related to basketball team (linked by an orange dashed line) may make the global models mistakenly refer "France" to France national basketball team. So, how to solve these problems?

We note that, mentions in text usually have different disambiguation difficulty according to the quality of contextual information and the topical coherence. Intuitively, if we start with mentions that are easier to disambiguate and gain correct results, it will be effective to utilize information provided by previously referred entities to disambiguate subsequent mentions. In the above example, it is much easier to map "2018 World Cup" to 2018 FIFA World Cup 


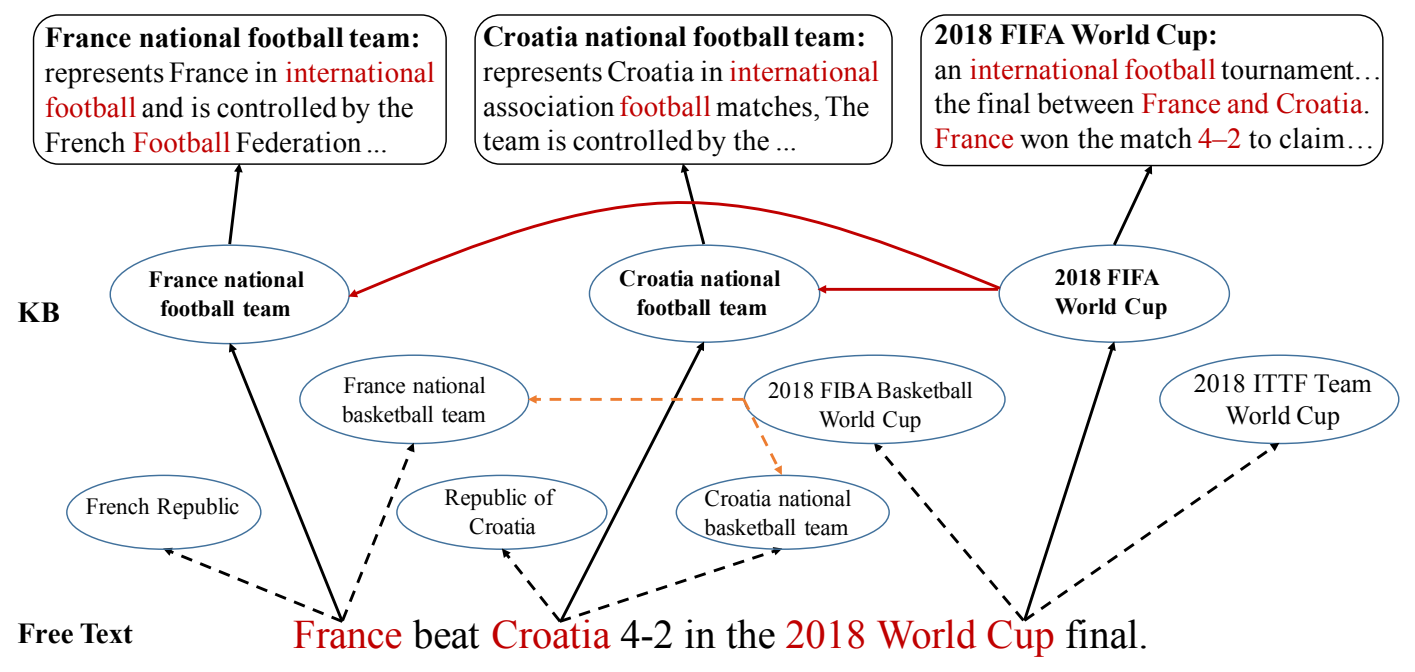

Figure 1: Illustration of mentions in the free text and their candidate entities in the knowledge base. Solid black lines point to the correct target entities corresponding to the mentions and to the descriptions of these correct target entities. Solid red lines indicate the consistency between correct target entities and the orange dashed lines denote the consistency between wrong candidate entities.

based on their common contextual words "France", "Croatia", "4-2". Then, it is obvious that "France" and "Croatia" should be referred to the national football team because football-related terms are mentioned many times in the description of 2018 FIFA World Cup.

Inspired by this intuition, we design the solution with three principles: (i) utilizing local features to rank the mentions in text and deal with them in a sequence manner; (ii) utilizing the information of previously referred entities for the subsequent entity disambiguation; (iii) making decisions from a global perspective to avoid the error propagation if the previous decision is wrong.

In order to achieve these aims, we consider global EL as a sequence decision problem and propose a deep reinforcement learning (RL) based model, RLEL for short, which consists of three modules: Local Encoder, Global Encoder and Entity Selector. For each mention and its candidate entities, Local Encoder encodes the local features to obtain their latent vector representations. Then, the mentions are ranked according to their disambiguation difficulty, which is measured by the learned vector representations. In order to enforce global coherence between mentions, Global Encoder encodes the local representations of mention-entity pairs in a sequential manner via a LSTM network, which maintains a long-term memory on features of entities which has been selected in previous states. Entity Selector uses a policy network to choose the target entities from the candidate set. For a single disambiguation decision, the policy network not only considers the pairs of current mention-entity representations, but also concerns the features of referred entities in the previous states which is pursued by the Global Encoder. In this way, Entity Selector is able to take actions based on the current state and previous ones. When eliminating the ambiguity of all mentions in the sequence, delayed rewards are used to adjust its policy in order to gain an optimized global decision.
Deep RL model, which learns to directly optimize the overall evaluation metrics, works much better than models which learn with loss functions that just evaluate a particular single decision. By this property, RL has been successfully used in many NLP tasks, such as information retrieval [28], dialogue system [10] and relation classification [12], etc. To the best of our knowledge, we are the first to design a RL model for global entity linking. And in this paper, our RL model is able to produce more accurate results by exploring the long-term influence of independent decisions and encoding the entities disambiguated in previous states.

In summary, the main contributions of our paper mainly include following aspects:

- We are the first to consider EL as a sequence decision problem and innovatively utilize a deep reinforcement learning model in this task.

- The proposed model takes into account both local context and global coherence. In the process of global disambiguation, we make full use of the previous selected entity information and make decisions from a global perspective.

- We evaluate our model on several benchmark datasets and the experimental results show that our model achieves significant improvements over the state-of-the-art methods.

\section{METHODOLOGY}

The overall structure of our RLEL model is shown in Figure 2. The proposed framework mainly includes three parts: Local Encoder which encodes local features of mentions and their candidate entities, Global Encoder which encodes the global coherence of mentions in a sequence manner and Entity Selector which selects an entity from the candidate set. In the training process, Local Encoder is trained independently because its output is just used as the input of Entity Selector. Entity Selector and Global Encoder are 


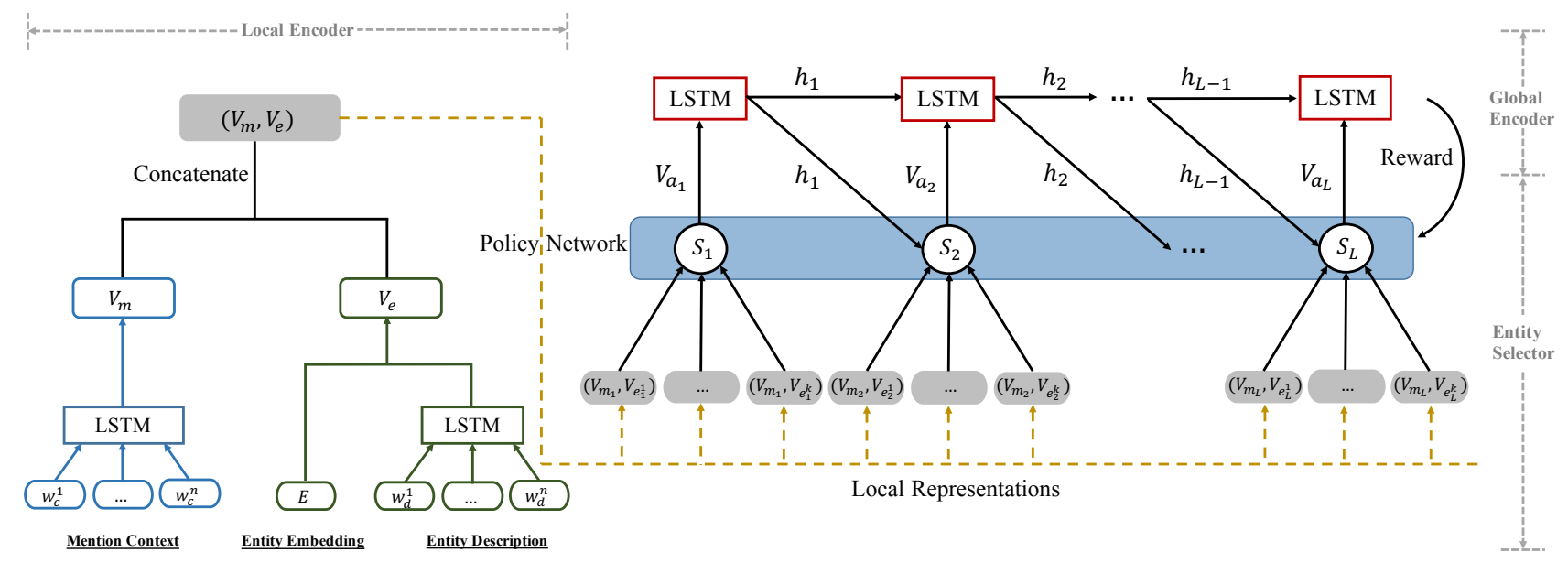

Figure 2: The overall structure of our RLEL model. It contains three parts: Local Encoder, Global Encoder and Entity Selector. In this framework, $\left(V_{m_{t}}, V_{\boldsymbol{e}_{t}^{k}}\right)$ denotes the concatenation of the mention context vector $V_{m_{t}}$ and one candidate entity vector $V_{e_{t}^{k}}$. The policy network selects one entity from the candidate set, and $V_{a_{t}}$ denotes the concatenation of the mention context vector $V_{m_{t}}$ and the selected entity vector $V_{e_{t}^{*}}$. $h_{t}$ represents the hidden status of $V_{a_{t}}$, and it will be fed into $S_{t+1}$.

trained jointly, because these two modules interact on each other. In the following, we will introduce the technical details of these modules.

\subsection{Preliminaries}

Before introducing our model, we firstly define the entity linking task. Formally, given a document $D$ with a set of mentions $M=\left\{m_{1}, m_{2}, \ldots, m_{k}\right\}$, each mention $m_{t} \in D$ has a set of candidate entities $C_{m_{t}}=\left\{e_{t}^{1}, e_{t}^{2}, \ldots, e_{t}^{n}\right\}$. The task of entity linking is to map each mention $m_{t}$ to its corresponding correct target entity $e_{t}^{+}$or return "NIL" if there is no correct target entity in the knowledge base. Before selecting the target entity, we need to generate a certain number of candidate entities for model selection.

Inspired by the previous works [29, 31, 42], we use the mention's redirect and disambiguation pages in Wikipedia to generate candidate sets. For those mentions without corresponding disambiguation pages, we use its n-grams to retrieve the candidates [31] In most cases, the disambiguation page contains many, sometimes even hundreds of entities. To optimize the model's memory and avoid unnecessary calculations, the candidate sets need to be filtered $[1,14,37]$. Here we utilize the XGBoost model [3] as an entity ranker, in which the useful features can be divided into two aspects, the one is string similarity such as the Jaro-Winkler distance between the entity title and the mention, the other is semantic similarity such as the cosine distance between the mention context representation and the entity embedding. Furthermore, we also use the statistical features based on the pageview and hyperlinks in Wikipedia. Empirically, we get the pageview of the candidate entities from the Wikipedia Tool Labs ${ }^{1}$ which counts the number of visits on each entity page in Wikipedia. After ranking the candidates based on above features, we take the top $k$ entities as final candidate set for each mention.

\footnotetext{
${ }^{1}$ The url of the website is: https://tools.wmflabs.org/pageviews/
}

\subsection{Local Encoder}

Given a mention $m_{t}$ and the corresponding candidate set $\left\{e_{t}^{1}, e_{t}^{2}, \ldots\right.$, $\left.e_{t}^{k}\right\}$, we aim to get their local representation based on the mention context and the candidate entity description. For each mention, we firstly select its $n$ surrounding words, and represent them as word embedding using a pre-trained lookup table [24]. Then, we use Long Short-Term Memory (LSTM) networks to encode the contextual word sequence $\left\{w_{c}^{1}, w_{c}^{2}, \ldots, w_{c}^{n}\right\}$ as a fixed-size vector $V_{m_{t}}$. The description of entity is encoded as $D_{e_{t}^{i}}$ in the same way. Apart from the description of entity, there are many other valuable information in the knowledge base. To make full use of these information, many researchers trained entity embeddings by combining the description, category, and relationship of entities. As shown in [14], entity embeddings compress the semantic meaning of entities and drastically reduce the need for manually designed features or co-occurrence statistics. Therefore, we use the pre-trained entity embedding $E_{e_{t}^{i}}$ and concatenate it with the description vector $D_{e_{t}^{i}}$ to enrich the entity representation. The concatenation result is denoted by $V_{e_{t}^{i}}$.

After getting $V_{e_{t}^{i}}$, we concatenate it with $V_{m_{t}}$ and then pass the concatenation result to a multilayer perceptron (MLP). The MLP outputs a scalar to represent the local similarity between the mention $m_{t}$ and the candidate entity $e_{t}^{i}$. The local similarity is calculated by the following equations:

$$
\Psi\left(m_{t}, e_{t}^{i}\right)=\operatorname{MLP}\left(V_{m_{t}} \oplus V_{e_{t}^{i}}\right)
$$

Where $\oplus$ indicates vector concatenation. With the purpose of distinguishing the correct target entity and wrong candidate entities when training the local encoder model, we utilize a hinge loss that ranks ground truth higher than others. The rank loss function is defined as follows:

$$
L_{\text {local }}=\max \left(0, \gamma-\Psi\left(m_{t}, e_{t}^{+}\right)+\Psi\left(m_{t}, e_{t}^{-}\right)\right)
$$


When optimizing the objective function, we minimize the rank loss according to [14, 37]. In this ranking model, a training instance is constructed by pairing a positive target entity $e_{t}^{+}$with a negative entity $e_{t}^{-}$, where $\gamma>0$ is a margin parameter. Our purpose is to make the score of the positive target entity $e_{t}^{+}$be at least a margin $\gamma$ higher than that of negative candidate entity $e_{t}^{-}$.

With the local encoder, we obtain the representation of mention context and candidate entities, which will be used as the input of the entity selector. In addition, the similarity scores calculated by MLP will be utilized for ranking mentions in the global encoder.

\subsection{Global Encoder}

In the global encoder module, we aim to enforce the topical coherence among the mentions and their target entities. So, we use an LSTM network which is capable of maintaining the long-term memory to encode the ranked mention sequence. What we need to emphasize is that our global encoder just encode the mentions that have been disambiguated by the entity selector which is denoted as $V_{a_{t}}$.

As mentioned above, the mentions should be sorted according to their contextual information and topical coherence. So, we firstly divide all mentions into several segments by the order they appear in the document based on the observation that the topical consistency attenuates along with the distance between the mentions. Then, we sort mentions in a segment from highest to lowest local similarity. As shown in Equation 1, the local similarity of $m_{i}$ and its corresponding candidate entity $e_{i}^{t}$ is defined as $\Psi\left(m_{i}, e_{i}^{t}\right)$. On this basis, we define $\Psi_{\max }\left(m_{i}, e_{i}^{a}\right)$ as the the maximum local similarity between $m_{i}$ and its candidate set $C_{m_{i}}=\left\{e_{i}^{1}, e_{i}^{2}, \ldots, e_{i}^{n}\right\}$. The $\Psi_{\max }\left(m_{i}, e_{i}^{a}\right)$ is used as criterion when sorting mentions. For instance, if $\Psi_{\max }\left(m_{i}, e_{i}^{a}\right)>\Psi_{\max }\left(m_{j}, e_{j}^{b}\right)$, we place $m_{i}$ before $m_{j}$. In this order, global encoder memorizes each selected entity and the encoding result will be served as input to the entity selector. Based on the topical coherence between the selected entities, entity selector will perform mention disambiguation from the global perspective.

Before using entity selector to choose target entities, we pretrain the global LSTM network. During the training process, we input not only positive samples but also negative ones to the LSTM. By doing this, we can enhance the robustness of the global encoder module. To pre-train this module, we adopt the following cross entropy loss function .

$$
L_{\text {global }}=-\frac{1}{n} \sum_{x}\left[y \ln y^{\prime}+(1-y) \ln \left(1-y^{\prime}\right)\right]
$$

Where $y \in\{0,1\}$ represents the real label of the candidate entity. If the candidate entity is correct, $y=1$; otherwise $y=0 . y^{\prime} \in(0,1)$ indicates the predicted result of our model. After pre-training the global encoder, we use the entity selector to choose the target entity for each mention and encode these selections.

\subsection{Entity Selector}

The entity selector module aims to select the target entity from the candidates based on the results of local and global encoder. In the process of sequence disambiguation, each selection will have an impact on subsequent decisions. Therefore, we convert the global

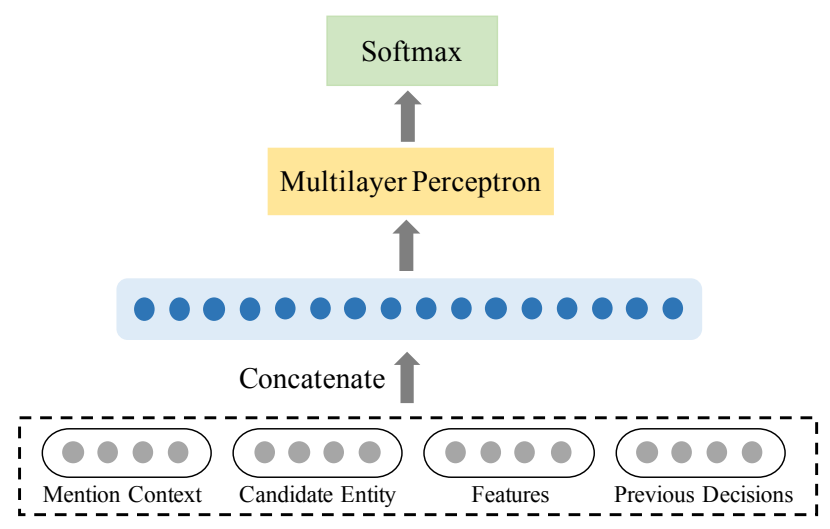

Figure 3: The architecture of policy network. It is a feedforward neural network and the input consists of four parts: mention context representation, candidate entity representation, feature representation, and encoding of the previous decisions.

entity disambiguation into a reinforcement learning problem and consider the entity selector as an agent. In our model, the agent is designed as a policy network which can learn a stochastic policy and prevents the agent from getting stuck at an intermediate state [39]. Under the guidance of policy, the agent decides which action (choosing one target entity from the candidates) should be taken at each state (current local and global encoding), and will receive a delay reward after all decisions are made. In the following, we will introduce the state, action and reward, and detail how to select target entity via a policy network.

State. The result of entity selection is based on the current state information. For time $t$, the state vector $S_{t}$ is generated as follows:

$$
S_{t}=V_{m_{i}}^{t} \oplus V_{e_{i}}^{t} \oplus V_{\text {feature }}^{t} \oplus V_{e^{*}}^{t-1}
$$

Where $\oplus$ indicates vector concatenation. $V_{m_{i}}^{t}$ and $V_{e_{i}}^{t}$ respectively denote the vector of $m_{i}$ and $e_{i}$ at time $t$. For each mention, there are $k$ candidate entities. In order to compare the semantic relevance between the mention and each candidate entity, we use multiple copies of the mention vector. Formally, we extend $V_{m_{i}}^{t} \in \mathbb{R}^{1 \times n}$ to $V_{m_{i}}{ }^{\prime} \in \mathbb{R}^{k \times n}$ and then combine it with $V_{e_{i}}^{t} \in \mathbb{R}^{k \times n}$. Since $V_{m_{i}}^{t}$ and $V_{e_{i}}^{t}$ mainly represent semantic information, we add feature vector $V_{\text {feature }}^{t}$ to enrich lexical and statistical features. These features mainly include the popularity of the entity, the edit distance between the entity description and the mention context, the number of identical words in the entity description and the mention context, etc. In addition, the global feature $V_{e^{*}}^{t-1}$ is also added to $S_{t}$. As mentioned in global encoder module, $V_{e^{*}}^{t-1}$ is the output of the global LSTM network at time $t-1$, which encodes the mention context and the selected entity from time 0 to $t-1$. Thus, the state $S_{t}$ consists of current information and previous decisions, containing the semantic features and statistical ones. Next, the concatenated vector will be fed into the policy network to generate action.

Action. According to the status at each time step, we take corresponding action. Specifically, we define the action at time step $t$ is to 
select the target entity $e_{t}^{*}$ for $m_{t}$. The size of action space is the number of candidate entities for each mention, where $a_{i} \in\{0,1,2 \ldots k\}$ indicates the position of the selected entity in the candidate list. After performing all actions in the sequence we will get a delayed reward.

Reward. The agent takes the reward value as the feedback of its actions and learns the policy based on it. Since one selection has a long-term impact on subsequent decisions, we give a delay reward rather than an immediate one, reflecting whether the action improves the overall performance or not.

$$
R\left(a_{t}\right)=p\left(a_{t}\right) \sum_{j=t}^{T} p\left(a_{j}\right)+\left(1-p\left(a_{t}\right)\right)\left(\sum_{j=t}^{T} p\left(a_{j}\right)+t-T\right)
$$

where $p\left(a_{t}\right) \in\{0,1\}$ indicates whether the current action is correct or not. If the action is correct, $p\left(a_{t}\right)=1$; otherwise, $p\left(a_{t}\right)=0$. Hence $\sum_{j=t}^{T} p\left(a_{j}\right)$ and $\sum_{j=t}^{T} p\left(a_{j}\right)+t-T$ respectively represent the number of correct actions and that of wrong ones from time $t$ to the end of episode. Based on the above definition, our delayed reward can be used to guide the learning of the policy for entity linking.

Policy Network. After defining the state, action and reward, our main challenge is how to choose an action from the action space. To solve this problem, we sample the value of each action using a policy network $\pi_{\Theta}(a \mid s)$. The structure of the policy network is shown in Figure 3. The input of the network is the current state, including the mention context representation, candidate entity representation, feature representation, and encoding of the previous decisions. We concatenate these representations and fed them into a multilayer perceptron. For each hidden layer, we generate the output by:

$$
h_{i}\left(S_{t}\right)=\operatorname{Relu}\left(W_{i} * h_{i-1}\left(S_{t}\right)+b_{i}\right)
$$

Where $W_{i}$ and $b_{i}$ are the parameters of the $i$ th hidden layer, and we use Relu as the activation function. After getting the output of the last hidden layer, we feed it into a softmax layer which generates the probability distribution of actions. The probability distribution is generated as follows:

$$
\pi(a \mid s)=\operatorname{Softmax}\left(W * h_{l}(S)+b\right)
$$

Where the $W$ and $b$ are the parameters of the softmax layer. For each mention in the sequence, we take one action to select the target entity from its candidate set. After performing all decisions in the episode, each action will get an expected reward and our goal is to maximize the expected total rewards. Formally, the objective function is defined as:

$$
\begin{aligned}
J(\Theta) & =\mathbb{E}_{\left(s_{t}, a_{t}\right) \sim P_{\Theta}\left(s_{t}, a_{t}\right)} R\left(s_{1} a_{1} \ldots s_{L} a_{L}\right) \\
& =\sum_{t} \sum_{a} \pi_{\Theta}(a \mid s) R\left(a_{t}\right)
\end{aligned}
$$

Where $P_{\Theta}\left(s_{t}, a_{t}\right)$ is the state transfer function, $\pi_{\Theta}(a \mid s)$ indicates the probability of taking action $a$ under the state $s$, and $R\left(a_{t}\right)$ is the expected reward of action $a$ at time step $t$. According to REINFORCE policy gradient algorithm[38], we update the policy gradient by the equation 9.

$$
\Theta \leftarrow \Theta+\alpha \sum_{t} R\left(a_{t}\right) \nabla_{\Theta} \log \pi_{\Theta}(a \mid s)
$$

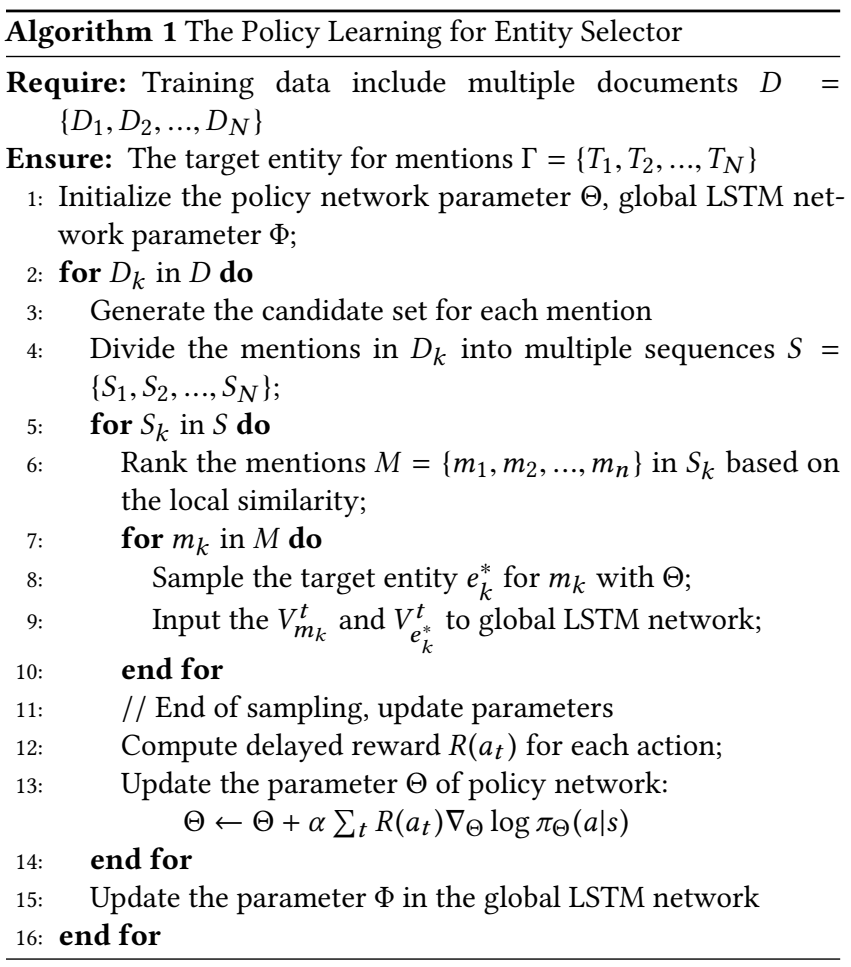

As the global encoder and the entity selector affect each other, we train them jointly after pre-training the two networks. The details of the joint learning are presented in Algorithm 1.

\section{EXPERIMENT}

In order to evaluate the effectiveness of our method, we train the RLEL model and validate it on a series of popular datasets that are also used by [14, 37]. To avoid overfitting with one dataset, we use both AIDA-Train and Wikipedia data in the training set. Furthermore, we compare the RLEL with some baseline methods, and our model achieves the state-of-the-art results. We implement our models in Tensorflow and run experiments on 4 Tesla V100 GPU.

\subsection{Experiment Setup}

Datasets. We conduct experiments on several different types of public datasets including news and encyclopedia corpus. The training set is AIDA-Train and Wikipedia datasets, where AIDA-Train contains 18448 mentions and Wikipedia contains 25995 mentions. In order to compare with the previous methods, we evaluate our model on AIDA-B and other datasets. These datasets are well-known and have been used for the evaluation of most existing entity linking systems. The statistics of the datasets are shown in Table 1 .

- AIDA-CoNLL [20] is annotated on Reuters news articles. It contains training (AIDA-Train), validation (AIDA-A) and test (AIDA-B) sets.

- ACE2004 [34] is a subset of the ACE2004 Coreference documents.

- MSNBC [9] contains top two stories in the ten news categories(Politics, Business, Sports etc.) 
Table 1: Statistics of document and mention numbers on experimental datasets.

\begin{tabular}{|c|c|c|c|}
\hline Dataset & Doc Num & Mention Num & Mentions Per Doc \\
\hline AIDA-Train & 946 & 18448 & 19.5 \\
AIDA-A & 216 & 4791 & 22.1 \\
AIDA-B & 231 & 4485 & 19.4 \\
\hline ACE2004 & 36 & 251 & 7.1 \\
\hline MSNBC & 20 & 656 & 32.8 \\
\hline AQUAINT & 50 & 727 & 14.5 \\
\hline WNED-CWEB & 320 & 11154 & 34.8 \\
\hline WNED-WIKI & 320 & 6821 & 21.3 \\
\hline OURSELF-WIKI & 460 & 25995 & 56.5 \\
\hline
\end{tabular}

- AQUAINT [25] is a news corpus from the Xinhua News Service, the New York Times, and the Associated Press.

- WNED-CWEB [16] is randomly picked from the FACC1 annotated ClueWeb 2012 dataset.

- WNED-WIKI [16] is crawled from Wikipedia pages with its original hyperlink annotation.

- OURSELF-WIKI is crawled by ourselves from Wikipedia pages.

Training Details. During the training of our RLEL model, we select top $k$ candidate entities for each mention to optimize the memory and run time. In the top $k$ candidate list, we define the recall of correct target entity is $R_{t}$. According to our statistics, when $k$ is set to $1,5,10, R_{t}$ is $0.853,0.977,0.993$ respectively. Empirically, we choose top 5 candidate entities as the input of our RLEL model. For each candidate entity, in order to get the entity description information and reduce the impact of noise, we use TextRank algorithm [23] to select 15 keywords from its original description in wikipedia. Similarly, we choose 15 words around the mention as its context. In the global LSTM network, if the number of mentions does not reach the pre-defined sequence length, we adopt the mention padding strategy. In short, we use the last mention to pad the sequence until the number of mentions reaches the length.

Hyper-parameter setting. We set the dimensions of word embedding and entity embedding to 300 , where the word embedding and entity embedding are released by [30] and [14] respectively. For parameters of the local LSTM network, the number of LSTM cell units is set to 512 , the batch size is 64 , and the rank margin $\gamma$ is 0.1 . Similarly, in global LSTM network, the number of LSTM cell units is 700 and the batch size is 16. In the above two LSTM networks, the learning rate is set to $1 \mathrm{e}-3$, the probability of dropout is set to 0.8 , and the Adam is utilized as optimizer. In the policy network, we set the number of MLP layers to 4 and extend the priori feature dimension to 50 .

\subsection{Comparing with Previous Work}

Baselines. We compare RLEL with a series of EL systems which report state-of-the-art results on the test datasets. There are various methods including classification model [25], rank model [6, 34] and probability graph model [14, 16, 20, 21, 37]. Besides, Cheng et al.[5] formulate their global decision problem as an Integer Linear Program (ILP) which incorporates the entity-relation inference.
Table 2: In-KB accuracy result on AIDA-B dataset.

\begin{tabular}{|c|c|}
\hline Methods & AIDA-B \\
\hline Huang and Heck (2015)[21] & $86.6 \%$ \\
\hline Chisholm and Hachey (2015)[6] & $88.7 \%$ \\
\hline Guo and Barbosa (2016)[16] & $89.0 \%$ \\
\hline Globerson et al. (2016)[15] & $91.0 \%$ \\
\hline Yamada et al. $(2016)[40]$ & $91.5 \%$ \\
\hline Ganea and Hofmann (2017)[14] & $92.2 \%$ \\
\hline Phong and Titov (2018)[37] & $93.1 \%$ \\
\hline our & $\mathbf{9 4 . 3 \%}$ \\
\hline
\end{tabular}

Globerson et al. [15] introduce a multi-focal attention model which allows each candidate to focus on limited mentions, Yamada et al.[40] propose a word and entity embedding model specifically designed for EL.

Evaluation Metric. We use the standard Accuracy, Precision, Recall and F1 at mention level (Micro) as the evaluation metrics:

$$
\begin{gathered}
\text { Accuracy }=\frac{\left|M \cap M^{*}\right|}{\left|M \cup M^{*}\right|} \\
\text { Precision }=\frac{\left|M \cap M^{*}\right|}{|M|} \\
\text { Recall }=\frac{\left|M \cap M^{*}\right|}{\left|M^{*}\right|} \\
F 1=\frac{2 * \text { Precision } * \text { Recall }}{\text { Precision }+ \text { Recall }}
\end{gathered}
$$

where $M^{*}$ is the golden standard set of the linked name mentions, $M$ is the set of linked name mentions outputted by an EL method.

Results. As same as previous work, we use in-KB accuracy and micro F1 to evaluate our method. We first test the model on the AIDA-B dataset. From Table 2, we can observe that our model achieves the best result. Previous best results on this dataset are generated by $[14,37]$ which both built CRF models. They calculate the pairwise scores between all candidate entities. Differently, our model only considers the consistency of the selected entities and ignores the relationship between incorrect candidates. The experimental results show that our model can reduce the impact of noise and improve the accuracy of disambiguation. Apart from experimenting on AIDA-B, we also conduct experiments on several different datasets to verify the generalization performance of our model.

From Table 3, we can see that RLEL has achieved relatively good performances on ACE2004, CWEB and WIKI. Previous models $[5,14,37]$ achieve better performances on the news datasets such as MSNBC and AQUINT, but their results on encyclopedia datasets such as WIKI are relatively poor. To avoid overfitting with some datasets and improve the robustness of our model, we not only use AIDA-Train but also add Wikipedia data to the training set. In the end, our model achieve the best overall performance.

In most existing EL systems, entities with lower frequency are difficult to disambiguate. For further analysis, we focus on the disambiguation accuracy of the gold entities in AIDA-B dataset with low popularity. We divide the gold entities according to their 
Table 3: Compare our model with other baseline methods on different types of datasets. The evaluation metric is micro F1.

\begin{tabular}{|c|c|c|c|c|c|c|}
\hline Methods & MSNBC & AQUAINT & ACE2004 & CWEB & WIKI & Avg \\
\hline Milne and Witten (2008)[25] & $78 \%$ & $85 \%$ & $81 \%$ & $64.1 \%$ & $81.7 \%$ & $77.96 \%$ \\
\hline Hoffart and Johannes(2011)[20] & $79 \%$ & $56 \%$ & $80 \%$ & $58.6 \%$ & $63 \%$ & $67.32 \%$ \\
\hline Ratinov and Lev[34] & $75 \%$ & $83 \%$ & $82 \%$ & $56.2 \%$ & $67.2 \%$ & $72.68 \%$ \\
\hline Cheng and Roth (2013)[5] & $90 \%$ & $\mathbf{9 0 \%}$ & $86 \%$ & $67.5 \%$ & $73.4 \%$ & $81.38 \%$ \\
\hline Guo and Barbosa (2016)[16] & $92 \%$ & $87 \%$ & $88 \%$ & $77 \%$ & $\mathbf{8 4 . 5 \%}$ & $85.7 \%$ \\
\hline Ganea and Hofmann (2017)[14] & $93.7 \%$ & $88.5 \%$ & $88.5 \%$ & $77.9 \%$ & $77.5 \%$ & $85.22 \%$ \\
\hline Phong and Titov (2018)[37] & $\mathbf{9 3 . 9 \%}$ & $88.3 \%$ & $89.9 \%$ & $77.5 \%$ & $78.0 \%$ & $85.51 \%$ \\
\hline our & $92.8 \%$ & $87.5 \%$ & $\mathbf{9 1 . 2 \%}$ & $\mathbf{7 8 . 5 \%}$ & $82.8 \%$ & $\mathbf{8 6 . 5 6 \%}$ \\
\hline
\end{tabular}

Table 4: The micro F1 of gold entities with different pageviews on part of AIDA-B dataset.

\begin{tabular}{|c|c|c|}
\hline Pageview/million & Mention Num & Micro F1 \\
\hline$<0.01$ & 307 & $91.93 \%$ \\
\hline $0.01-0.1$ & 612 & $86.06 \%$ \\
\hline $0.1-1$ & 968 & $88.97 \%$ \\
\hline $1-5$ & 1006 & $96.03 \%$ \\
\hline $5-10$ & 493 & $96.43 \%$ \\
\hline$>10$ & 825 & $99.39 \%$ \\
\hline
\end{tabular}

pageviews in wikipedia, and the statistical disambiguation results are shown in Table 4. Since some pageviews can not be obtained, we only count a part of gold entities. The result indicates that our model still works well for low-frequency entities. But for mediumfrequency gold entities, our model doesn't work well enough. The most important reason is that other candidate entities corresponding to these medium-frequency gold entities have higher pageviews and local similarities, which makes the model difficult to distinguish.

\subsection{Discussion on different RLEL variants}

To demonstrate the effects of RLEL, we evaluate our model under different conditions. First, we evaluate the effect of sequence length on global decision making. Secondly, we test whether sorting the mentions have a positive effect on the results. Thirdly, we analyze the results of not adding globally encoding during entity selection. Finally, we compare our RL selection strategy with the greedy choice.

Sequence in different length. A document may contain multiple topics, so we do not add all mentions to a single sequence. In practice, we add some adjacent mentions to the sequence and use reinforcement learning to select entities from beginning to end. To analyze the impact of the number of mentions on joint disambiguation, we experiment with sequences on different lengths. The results on AIDA-B are shown in Figure 4. We can see that when the sequence is too short or too long, the disambiguation results are both very poor. When the sequence length is less than 3, delay reward can't work in reinforcement learning, and when the sequence length reaches 5 or more, noise data may be added. Finally, we choose the 4 adjacent mentions to form a sequence.

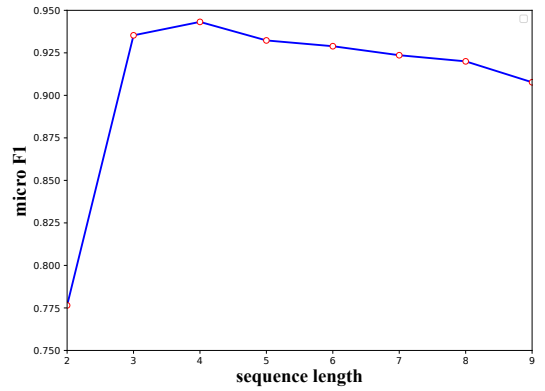

Figure 4: The performance of models with different sequence lengths on AIDA-B dataset.

Influence of ranking mentions. In this section, we test whether ranking mentions is helpful for entity selections. We directly input the mentions into the global encoder by the order they appear in the text. We record the disambiguation results and compare them with the method which adopts ranking mentions. As shown in Figure 5a, the model with ranking mentions achieved better performances on most of datasets, indicating that it is effective to place the mention that with a higher local similarity in front of the sequence. It is worth noting that the effect of ranking mentions is not obvious on the MSNBC dataset. The reason is that most of mentions in MSNBC have similar local similarities, so the order of disambiguation has little effect on the final result.

Effect of global encoding. Different from previous global methods which use pairwise similarity between entities to capture their correlation, our model encodes the selected entity information in sequence. To evaluate whether the global encoding contributes to disambiguation, we compare the performance between the model with and without adding the global information. When the global encoding is not taken in to account, the current state only contains the local information, i.e., the mention context representation, candidate entity representation and feature representation. From the results in Figure 5b, we can see that the model with global encoding achieves an improvement of $4 \%$ accuracy over the model without global encoding.

Different entity selection strategies. To illustrate the effectiveness for adopting the reinforcement learning for entity selection, we compare two entity selection strategies like [12]. Specifically, we 


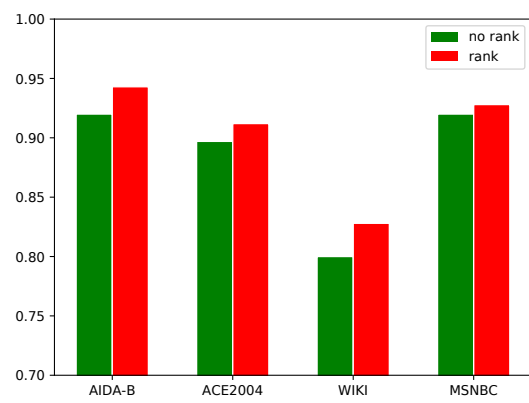

a.The influence of ranking mentions for entity selection.

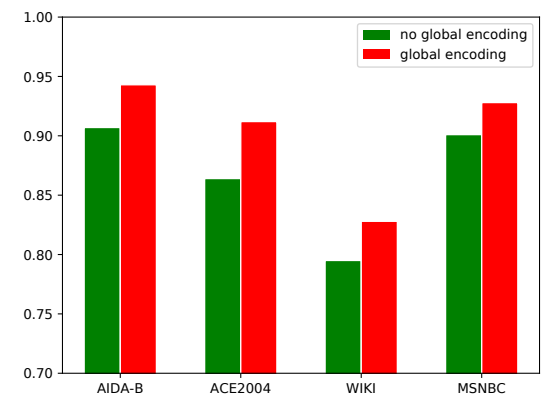

$b$. The effect of the global encoding for entity selection.

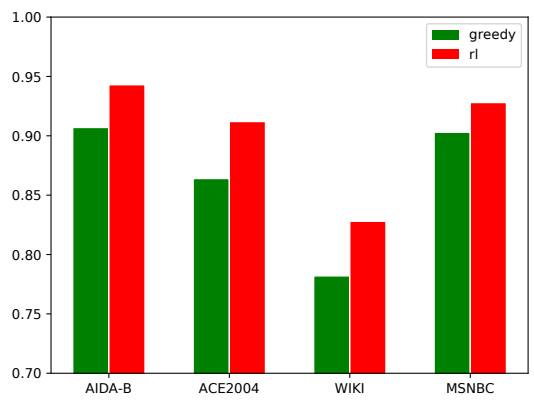

c. Comparison of reinforcement learning selection with greedy choice.

Figure 5: The comparative experiments of RLEL model.

Table 5: Entity selection examples by our RLEL model.

\begin{tabular}{|c|c|c|}
\hline Document Content & Mentions after ranking & Selected Target Entity(is correct) \\
\hline $\begin{array}{l}\text { Australia beat West Indies by five wickets } \\
\text { in a World Series limited overs match at the } \\
\text { Melbourne Cricket Groundon Friday... }\end{array}$ & $\begin{array}{l}\text { 1.Melbourne Cricket Ground } \\
\text { 2.World Series } \\
\text { 3.West Indies } \\
\text { 4.Australia }\end{array}$ & $\begin{array}{l}\text { 1.Melbourne Cricket Ground(correct) } \\
\text { 2.World Series Cricket(correct) } \\
\text { 3.West Indies cricket team(correct) } \\
\text { 4.Australia national cricket team(correct) }\end{array}$ \\
\hline $\begin{array}{l}\text { Instead of Los Angeles International, } \\
\text {..., consider flying into Burbank or John } \\
\text { Wayne Airport in Orange County, Calif... }\end{array}$ & $\begin{array}{l}\text { 1.John Wayne Airport } \\
\text { 2.Orange County } \\
\text { 3.Los Angeles International } \\
\text { 4.Burbank }\end{array}$ & $\begin{array}{l}\text { 1.John Wayne Airport(correct) } \\
\text { 2.Orange County, California(correct) } \\
\text { 3.Los Angeles International Airport(correct) } \\
\text { 4.Burbank, California } \\
\text { (wrong, the correct is "Hollywood Burbank Airport") }\end{array}$ \\
\hline
\end{tabular}

perform entity selection respectively with reinforcement learning and greedy choice. The greedy choice is to select the entity with the largest local similarity from candidate set. But the reinforcement learning selection is guided by delay reward, which has a global perspective. In the comparative experiment, we just replace the RL selection with a greedy choice, keeping the other conditions consistent. From the results in Figure 5c, we can draw a conclusion that our entity selector performs much better than greedy strategies.

\subsection{Case Study}

Table 5 shows two entity selection examples by our RLEL model. For multiple mentions appearing in the document, we first sort them according to their local similarities, and select the target entities in order by the reinforcement learning model. From the results of sorting and disambiguation, we can see that our model is able to utilize the topical consistency between mentions and make full use of the selected target entity information.

\section{RELATED WORK}

\subsection{Entity Linking}

Entity linking can be roughly divided into two major categories: local and global disambiguation. Early studies use local models to resolve mentions independently, they usually disambiguate mentions based on lexical matching between the mention's surrounding words and the entity profile in the reference KB. Various methods have been proposed to model mention's local context ranging from binary classification [25] to rank models [4, 11]. In these methods, a large number of hand-designed features are applied. For some marginal mentions which are difficult to extract features, researchers also exploit the data retrieved by search engines $[7,8]$ or Wikipedia sentences [36]. However, the feature engineering and search engine based methods are both time-consuming and laborious. Recently, with the popularity of deep learning models, representation learning is utilized to automatically extract semantic features $[2,17]$. The entity representations learned by jointly modeling textual contexts and knowledge base are effective in combining multiple sources of information. To make full use of the information contained in representations, we also utilize the pre-trained entity embeddings in our model.

In recent years, with the assumption that the target entities of all mentions in a document shall be related, many novel global models for joint linking are proposed. Assuming the topical coherence among mentions, authors in $[13,33]$ construct factor graph models, which represent the mention and candidate entities as variable nodes, and exploit factor nodes to denote a series of features. Two recent studies $[14,37]$ use fully-connected pairwise Conditional Random Field(CRF) model and exploit loopy belief propagation to estimate the max-marginal probability. Moreover, PageRank or Random Walk [16, 19, 42] are utilized to select the target entity 
for each mention. The above probabilistic models usually need to predefine a lot of features and are difficult to calculate the maxmarginal probability as the number of nodes increases. In order to automatically learn features from the data, Cao et al. [1] applies Graph Convolutional Network to flexibly encode entity graphs. However, the graph-based methods are computationally expensive because there are lots of candidate entity nodes in the graph.

To reduce the calculation between candidate entity pairs, Globerson et al. [15] proposed a coherence model with an attention mechanism, in which each mention only focuses on a fixed number of mentions. Unfortunately, choosing the number of attention mentions is not easy in practice. Two recent studies [31, 32] finish linking all mentions by scanning the pairs of mentions at most once, they assume each mention only needs to be consistent with one another mention in the document. The limitation of their method is that the consistency information is too sparse, resulting in low confidence. Similar to us, Guo et al. [16] also sort mentions according to the difficulty of disambiguation, but they did not make full use of the information of previously referred entities for the subsequent entity disambiguation. Nguyen et al. [27] use the sequence model, but they simply encode the results of the greedy choice, and measure the similarities between the global encoding and the candidate entity representations. Their model does not consider the long-term impact of current decisions on subsequent choices, nor does they add the selected target entity information to the current state to help disambiguation.

\subsection{Reinforcement Learning}

In the last few years, reinforcement learning has emerged as a powerful tool for solving complex sequential decision-making problems. It is well known for its great success in the game field, such as Go [35] and Atari games [26]. Recently, reinforcement learning has also been successfully applied to many natural language processing tasks and achieved good performance [12, 22, 39]. Feng et al.[12] used reinforcement learning for relation classification task by filtering out the noisy data from the sentence bag and they achieved huge improvements compared with traditional classifiers. Zhang et al. [41] applied the reinforcement learning on sentence representation by automatically discovering task-relevant structures. To implement automatic taxonomy induction from a set of terms, Han et al. [18] designed an end-to-end reinforcement learning model to determine which term to select and where to place it on the taxonomy, and this model effectively reduced the error propagation between two phases. Inspired by the above works, we also use reinforcement learning in our framework.

\section{CONCLUSIONS}

In this paper we consider entity linking as a sequence decision problem and present a reinforcement learning based model. Our model learns the policy on selecting target entities in a sequential manner and makes decisions based on current state and previous ones. By utilizing the information of previously referred entities, we can take advantage of global consistency to disambiguate mentions. For each selection result in the current state, it also has a longterm impact on subsequent decisions, which allows learned policy strategy has a global view. In experiments, we evaluate our method on AIDA-B and other well-known datasets, the results show that our system outperforms state-of-the-art solutions. In the future, we would like to construct an end-to-end model which simultaneously optimize mention ranking and mention disambiguation.

\section{ACKNOWLEDGMENTS}

This research is supported by the National Key Research and Development Program of China (No. 2018YFB1004703), the Beijing Municipal Science and Technology Project under grant (No.

Z181100002718004), and the National Natural Science Foundation of China grants(No. 61602466).

\section{REFERENCES}

[1] Yixin Cao, Lei Hou, Juanzi Li, and Zhiyuan Liu. 2018. Neural Collective Entity Linking. In Proceedings of the 27th International Conference on Computational Linguistics, COLING 2018, Santa Fe, New Mexico, USA, August 20-26, 2018. 675-686.

[2] Yixin Cao, Lifu Huang, Heng Ji, Xu Chen, and Juanzi Li. 2017. Bridge Text and Knowledge by Learning Multi-Prototype Entity Mention Embedding. In Proceedings of the 55th Annual Meeting of the Association for Computational Linguistics, ACL 2017, Vancouver, Canada, fuly 30 - August 4, Volume 1: Long Papers. 1623-1633.

[3] Tianqi Chen and Carlos Guestrin. 2016. XGBoost: A Scalable Tree Boosting System. In Proceedings of the 22nd ACM SIGKDD International Conference on Knowledge Discovery and Data Mining, San Francisco, CA, USA, August 13-17, 2016. 785-794.

[4] Zheng Chen and Heng Ji. 2011. Collaborative Ranking: A Case Study on Entity Linking. In Proceedings of the 2011 Conference on Empirical Methods in Natural Language Processing, EMNLP 2011, 27-31 July 2011, Fohn McIntyre Conference Centre, Edinburgh, UK, A meeting of SIGDAT, a Special Interest Group of the ACL. 771-781.

[5] Xiao Cheng and Dan Roth. 2013. Relational Inference for Wikification. In Proceedings of the 2013 Conference on Empirical Methods in Natural Language Processing, EMNLP 2013, 18-21 October 2013, Grand Hyatt Seattle, Seattle, Washington, USA, A meeting of SIGDAT, a Special Interest Group of the ACL. 1787-1796.

[6] Andrew Chisholm and Ben Hachey. 2015. Entity Disambiguation with Web Links. TACL 3 (2015), 145-156.

[7] Marco Cornolti, Paolo Ferragina, Massimiliano Ciaramita, Stefan Rüd, and Hinrich Schütze. 2016. A Piggyback System for Joint Entity Mention Detection and Linking in Web Queries. In Proceedings of the 25th International Conference on World Wide Web, WWW 2016, Montreal, Canada, April 11 - 15, 2016. 567-578.

[8] Marco Cornolti, Paolo Ferragina, Massimiliano Ciaramita, Hinrich Schütze, and Stefan Rüd. 2014. The SMAPH system for query entity recognition and disambiguation. In ERD'14, Proceedings of the First ACM International Workshop on Entity Recognition \& Disambiguation, Fuly 11, 2014, Gold Coast, Queensland, Australia. 25-30.

[9] Silviu Cucerzan. 2007. Large-Scale Named Entity Disambiguation Based on Wikipedia Data. In EMNLP-CoNLL 2007, Proceedings of the 2007 Joint Conference on Empirical Methods in Natural Language Processing and Computational Natural Language Learning, fune 28-30, 2007, Prague, Czech Republic. 708-716.

[10] Bhuwan Dhingra, Lihong Li, Xiujun Li, Jianfeng Gao, Yun-Nung Chen, Faisal Ahmed, and Li Deng. 2017. Towards End-to-End Reinforcement Learning of Dialogue Agents for Information Access. In Proceedings of the 55th Annual Meeting of the Association for Computational Linguistics, ACL 2017, Vancouver, Canada, fuly 30 - August 4, Volume 1: Long Papers. 484-495.

[11] Mark Dredze, Paul McNamee, Delip Rao, Adam Gerber, and Tim Finin. 2010. Entity Disambiguation for Knowledge Base Population. In COLING 2010, 23rd International Conference on Computational Linguistics, Proceedings of the Conference, 23-27 August 2010, Beijing, China. 277-285.

[12] Jun Feng, Minlie Huang, Li Zhao, Yang Yang, and Xiaoyan Zhu. 2018. Reinforcement Learning for Relation Classification From Noisy Data. In Proceedings of the Thirty-Second AAAI Conference on Artificial Intelligence, New Orleans, Louisiana, USA, February 2-7, 2018.

[13] Octavian-Eugen Ganea, Marina Ganea, Aurélien Lucchi, Carsten Eickhoff, and Thomas Hofmann. 2016. Probabilistic Bag-Of-Hyperlinks Model for Entity Linking. In Proceedings of the 25th International Conference on World Wide Web, WWW 2016, Montreal, Canada, April 11 - 15, 2016. 927-938.

[14] Octavian-Eugen Ganea and Thomas Hofmann. 2017. Deep Joint Entity Disambiguation with Local Neural Attention. In Proceedings of the 2017 Conference on Empirical Methods in Natural Language Processing, EMNLP 2017, Copenhagen, Denmark, September 9-11, 2017. 2619-2629.

[15] Amir Globerson, Nevena Lazic, Soumen Chakrabarti, Amarnag Subramanya, Michael Ringgaard, and Fernando Pereira. 2016. Collective Entity Resolution with Multi-Focal Attention. In Proceedings of the 54th Annual Meeting of the 
Association for Computational Linguistics, ACL 2016, August 7-12, 2016, Berlin, Germany, Volume 1: Long Papers.

[16] Zhaochen Guo and Denilson Barbosa. 2018. Robust named entity disambiguation with random walks. Semantic Web 9, 4 (2018), 459-479.

[17] Nitish Gupta, Sameer Singh, and Dan Roth. 2017. Entity Linking via Joint Encoding of Types, Descriptions, and Context. In Proceedings of the 2017 Conference on Empirical Methods in Natural Language Processing, EMNLP 2017, Copenhagen, Denmark, September 9-11, 2017. 2681-2690.

[18] Jiawei Han, Xiang Ren, Jiaming Shen, Yuning Mao, and Xiaotao Gu. 2018. End-toEnd Reinforcement Learning for Automatic Taxonomy Induction. In Proceedings of the 56th Annual Meeting of the Association for Computational Linguistics, ACL 2018, Melbourne, Australia, fuly 15-20, 2018, Volume 1: Long Papers. 2462-2472.

[19] Xianpei Han, Le Sun, and Jun Zhao. 2011. Collective entity linking in web text: a graph-based method. In Proceeding of the 34th International ACM SIGIR Conference on Research and Development in Information Retrieval, SIGIR 2011, Beijing, China, July 25-29, 2011. 765-774.

[20] Johannes Hoffart, Mohamed Amir Yosef, Ilaria Bordino, Hagen Fürstenau, Manfred Pinkal, Marc Spaniol, Bilyana Taneva, Stefan Thater, and Gerhard Weikum. 2011. Robust Disambiguation of Named Entities in Text. In Proceedings of the 2011 Conference on Empirical Methods in Natural Language Processing, EMNLP 2011, 27-31 July 2011, John McIntyre Conference Centre, Edinburgh, UK, A meeting of SIGDAT, a Special Interest Group of the ACL. 782-792.

[21] Hongzhao Huang, Larry P. Heck, and Heng Ji. 2015. Leveraging Deep Neural Networks and Knowledge Graphs for Entity Disambiguation. CoRR abs/1504.07678 (2015). arXiv:1504.07678

[22] Ting Liu, William Yang Wang, Yu Zhang, Qingyu Yin, and Weinan Zhang. 2018 Deep Reinforcement Learning for Chinese Zero Pronoun Resolution. In Proceedings of the 56th Annual Meeting of the Association for Computational Linguistics, ACL 2018, Melbourne, Australia, fuly 15-20, 2018, Volume 1: Long Papers. 569-578.

[23] Rada Mihalcea and Paul Tarau. 2004. TextRank: Bringing Order into Text. In Proceedings of the 2004 Conference on Empirical Methods in Natural Language Processing , EMNLP 2004, A meeting of SIGDAT, a Special Interest Group of the ACL, held in conjunction with ACL 2004, 25-26 fuly 2004, Barcelona, Spain. 404-411.

[24] Tomas Mikolov, Kai Chen, Greg Corrado, and Jeffrey Dean. 2013. Efficient Estimation of Word Representations in Vector Space. CoRR abs/1301.3781 (2013) arXiv:1301.3781

[25] David N. Milne and Ian H. Witten. 2008. Learning to link with wikipedia. In Proceedings of the 17th ACM Conference on Information and Knowledge Management, CIKM 2008, Napa Valley, California, USA, October 26-30, 2008. 509-518.

[26] Volodymyr Mnih, K. Kavukcuoglu, D. Silver, A. A. Rusu, J. Veness, M. G. Bellemare, A. Graves, M. A. Riedmiller, A. Fidjeland, G. Ostrovski, S. Petersen, C. Beattie, A. Sadik, I. Antonoglou, H. King, D. Kumaran, D. Wierstra, S. Legg, and D. Hassabis. 2015. Human-level control through deep reinforcement learning. Nature 518, 7540 (2015), 529-533.

[27] Thien Huu Nguyen, Nicolas R. Fauceglia, Mariano Rodriguez-Muro, Oktie Hassanzadeh, Alfio Massimiliano Gliozzo, and Mohammad Sadoghi. 2016. Joint Learning of Local and Global Features for Entity Linking via Neural Networks. In COLING 2016, 26th International Conference on Computational Linguistics, Proceedings of the Conference: Technical Papers, December 11-16, 2016, Osaka, Japan. $2310-2320$.

[28] Rodrigo Nogueira and Kyunghyun Cho. 2017. Task-Oriented Query Reformulation with Reinforcement Learning. In Proceedings of the 2017 Conference on Empirical Methods in Natural Language Processing, EMNLP 2017, Copenhagen, Denmark, September 9-11, 2017. 574-583.

[29] Aasish Pappu, Roi Blanco, Yashar Mehdad, Amanda Stent, and Kapil Thadani 2017. Lightweight Multilingual Entity Extraction and Linking. In Proceedings of the Tenth ACM International Conference on Web Search and Data Mining, WSDM 2017, Cambridge, United Kingdom, February 6-10, 2017. 365-374.

[30] Jeffrey Pennington, Richard Socher, and Christopher D. Manning. 2014. Glove: Global Vectors for Word Representation. In Proceedings of the 2014 Conference on Empirical Methods in Natural Language Processing, EMNLP 2014, October 2529, 2014, Doha, Qatar, A meeting of SIGDAT, a Special Interest Group of the ACL. 1532-1543.

[31] Minh C. Phan, Aixin Sun, Yi Tay, Jialong Han, and Chenliang Li. 2017. NeuPL: Attention-based Semantic Matching and Pair-Linking for Entity Disambiguation. In Proceedings of the 2017 ACM on Conference on Information and Knowledge Management, CIKM 2017, Singapore, November 06 - 10, 2017. 1667-1676.

[32] Minh C. Phan, Aixin Sun, Yi Tay, Jialong Han, and Chenliang Li. 2018. PairLinking for Collective Entity Disambiguation: Two Could Be Better Than All. CoRR abs/1802.01074 (2018). arXiv:1802.01074

[33] Chenwei Ran, Wei Shen, and Jianyong Wang. 2018. An Attention Factor Graph Model for Tweet Entity Linking. In Proceedings of the 2018 World Wide Web Conference on World Wide Web, WWW 2018, Lyon, France, April 23-27, 2018. $1135-1144$.

[34] Lev-Arie Ratinov, Dan Roth, Doug Downey, and Mike Anderson. 2011. Local and Global Algorithms for Disambiguation to Wikipedia. In The 49th Annual Meeting of the Association for Computational Linguistics: Human Language Technologies, Proceedings of the Conference, 19-24 June, 2011, Portland, Oregon, USA. 1375-1384.

[35] David Silver, A. Huang, C. J. Maddison, A. Guez, L. Sifre, G. v. d. Driessche, J. Schrittwieser, I. Antonoglou, V. Panneershelvam, M. Lanctot, S. Dieleman, D. Grewe, J. Nham, N. Kalchbrenner, I. Sutskever, T. P. Lillicrap, M. Leach, K. Kavukcuoglu, T. Graepel, and D. Hassabis. 2016. Mastering the game of Go with deep neural networks and tree search. Nature 529, 7587 (2016), 484-489.

[36] Chuanqi Tan, Furu Wei, Pengjie Ren, Weifeng Lv, and Ming Zhou. 2017. Entity Linking for Queries by Searching Wikipedia Sentences. In Proceedings of the 2017 Conference on Empirical Methods in Natural Language Processing, EMNLP 2017, Copenhagen, Denmark, September 9-11, 2017. 68-77.

[37] Ivan Titov and Phong Le. 2018. Improving Entity Linking by Modeling Laten Relations between Mentions. In Proceedings of the 56th Annual Meeting of the Association for Computational Linguistics, ACL 2018, Melbourne, Australia, Fuly 15-20, 2018, Volume 1: Long Papers. 1595-1604.

[38] Ronald J. Williams. 1992. Simple Statistical Gradient-Following Algorithms for Connectionist Reinforcement Learning. Machine Learning 8 (1992), 229-256.

[39] Wenhan Xiong, Thien Hoang, and William Yang Wang. 2017. DeepPath: A Reinforcement Learning Method for Knowledge Graph Reasoning. In Proceedings of the 2017 Conference on Empirical Methods in Natural Language Processing, EMNLP 2017, Copenhagen, Denmark, September 9-11, 2017. 564-573.

[40] Ikuya Yamada, Hiroyuki Shindo, Hideaki Takeda, and Yoshiyasu Takefuji. 2016. Joint Learning of the Embedding of Words and Entities for Named Entity Disambiguation. In Proceedings of the 20th SIGNLL Conference on Computational Natural Language Learning, CoNLL 2016, Berlin, Germany, August 11-12, 2016. 250-259.

[41] Tianyang Zhang, Minlie Huang, and Li Zhao. 2018. Learning Structured Representation for Text Classification via Reinforcement Learning. In Proceedings of the Thirty-Second AAAI Conference on Artificial Intelligence, (AAAI-18), the 30th innovative Applications of Artificial Intelligence (IAAI-18), and the 8th AAAI Symposium on Educational Advances in Artificial Intelligence (EAAI-18), New Orleans, Louisiana, USA, February 2-7, 2018. 6053-6060.

[42] Stefan Zwicklbauer, Christin Seifert, and Michael Granitzer. 2016. Robust and Collective Entity Disambiguation through Semantic Embeddings. In Proceedings of the 39th International ACM SIGIR conference on Research and Development in Information Retrieval, SIGIR 2016, Pisa, Italy, fuly 17-21, 2016. 425-434. 